\title{
Composite Adenocarcinoma and Carcinoid Gastric Tumor in Chronic Atrophic Gastritis and Pernicious Anemia
}

\author{
Adam C. Adler ${ }^{\mathrm{a}}$ Cesar Cestero $^{\mathrm{a}} \quad$ Eugene H. Lewis III ${ }^{\mathrm{b}}$ \\ Ingram M. Roberts ${ }^{\mathrm{a}, \mathrm{c}}$ Eddy A. Castillo ${ }^{\mathrm{c}, \mathrm{d}}$ \\ Departments of a Internal Medicine and bPathology and Laboratory Medicine, and \\ 'Section of Gastroenterology, St. Vincent's Medical Center, Bridgeport, Conn.; \\ ${ }^{\mathrm{d}}$ Gastroenterology Associates of Fairfield County, Fairfield, Conn., USA
}

\section{Key Words}

Adenocarcinoma $\cdot$ Carcinoid $\cdot$ Collision tumor $\cdot$ Neuroendocrine $\cdot$ Stomach

\begin{abstract}
A 42-year-old Hispanic female was referred for investigation of unexplained weight loss. Initial upper endoscopy showed atrophic gastritis. Repeat endoscopy one year later revealed the presence of mixed composite tumor consisting of gastric adenocarcinoma and carcinoid tumors. Treatment was accomplished by surgical excision. Such cases are extremely rare and few such reports are available in the literature. We discuss the pathologies and means by which these tumors are classified and treated.
\end{abstract}

\section{Introduction}

Gastric cancer remains one of the leading causes of cancer-related deaths worldwide. The incidence of gastric neoplasm varies greatly throughout the world and calls into question the role of ethnicity, dietary and environmental impact on tumorigenesis [1]. Nearly $90 \%$ of gastric tumors are malignant, of which $95 \%$ are due to adenocarcinoma [1]. Gastric carcinoids, once considered a rare contributor to the aggregate group of gastrointestinal tumors, have been increasing in prevalence during recent years [2, 3]. It has been hypothesized that this increase in prevalence is owed to improved pathological methods for detection and a more aggressive approach to screening [4]. We present the case of an asymptomatic 42-year-old female who underwent routine upper endoscopy for anemia. Repeat endoscopy one year later revealed the presence of a composite tumor consisting of gastric adenocarcinoma and carcinoid tumors. 


\section{Gastric Carcinoids}

Carcinoid tumors are growths whose cellular components are derived from neuroendocrine cells. These tumors may or may not be in association with the carcinoid syndrome, depending on their production of active hormones, most notably the monoamine 5-hydroxytryptamine (5-HT or serotonin). There have been cases where carcinoid syndrome was found to be associated with the release of substrates such as epinephrine, norepinephrine, histamine, gastrin, adrenocorticotrophic hormone, melanocyte-stimulating hormone, calcitonin, vasoactive intestinal peptide, glucagon and somatostatin [5, 6]. Occasionally, immunohistologic analysis reveals the secretion of multiple hormones [6]. Carcinoid activity can be monitored by measurement of urinary 5-hydroxyindoleacetic acid, a byproduct of serotonin metabolism. Carcinoids can be subclassified based on the hormones produced, however the main determinants are cytoarchitectural components and specific immunohistochemical staining properties [5]. The appearance of carcinoid syndrome is dependent on the location of the tumor and occurs in locations where the venous drainage bypasses the liver's catabolic activities. Unfortunately, many gastric carcinoids are diagnosed rather late due to the absence of carcinoid syndrome $[3,5]$. Carcinoid tumors have been reported to occur in various organs throughout the body, with the most common locations being the jejunum, ileum, as well as the appendix and lungs. Carcinoids remain the most common primary neoplasm of the appendix. Although slow to progress, carcinoids may form metastatic lesions which are generally larger than their primary tumors, with a predilection for the mesenteric lymph nodes, liver and lung [6].

\section{Gastric Adenocarcinoma}

Adenocarcinoma is the most common type of histologic malignancy of gastric tissue. On occasion, gastric adenocarcinoma has been reported to coexist with other types of histologic malignancies found in the gastrointestinal tract, such as gastrointestinal stromal tumors, mucosal-associated lymphoid tissue and carcinoid tumors among others [7]. Concurrent existence of gastric adenocarcinoma with chronic atrophic gastritis is a well-documented entity [8]. Similarly, there has been documented evidence of increased occurrence of both gastric adenocarcinoma and gastric carcinoid tumors in patients with underlying pernicious anemia $[1,3,8,9]$. This is possibly due to metaplastic changes associated with chronic mucosal inflammation leading to the deposition of glandular cells. It is suggested that in patients with decreased acid secretion and thus increased gastrin levels, gastrin may serve as a trophic hormone by inducing hyperplasia of the neuroendocrine cells and inciting the formation of carcinoid tumor cells [10,11]. The treatment for gastric adenocarcinoma is surgery with either adjuvant or neoadjuvant therapy.

\section{Case Report}

We present the case of a 42-year-old female referred after experiencing a 6-pound weight loss the month prior. There was no nausea, vomiting, diarrhea, bloody stools or menorrhagia. The patient has a 10 -year history of pernicious anemia treated with intramuscular vitamin B12 injections. She is currently under treatment for Hashimoto's hypothyroidism and has a long history of chronic constipation. There was no history of alcohol, tobacco or drug use. She had a positive family history of colon carcinoma 


\begin{tabular}{|c|c|c|c|}
\hline $\begin{array}{r}\text { Case Reports in } \\
\text { castroenterolowy }\end{array}$ & $\begin{array}{l}\text { Case Rep Gastroenterol 2011;5:232-238 } \\
\text { DOI: } 10.1159 / 000327986\end{array}$ & $\begin{array}{l}\text { Published online: } \\
\text { April 15, } 2011\end{array}$ & \begin{tabular}{|l} 
(@) 2011 S. Karger AG, Basel \\
ISSN $1662-0631$ \\
www.karger.com/crg
\end{tabular} \\
\hline
\end{tabular}

from her father. Physical examination was unremarkable. Laboratory examination from the referring physician unavailable.

She was evaluated with upper endoscopy for identification of the cause for her iron deficiency anemia. Endoscopy revealed a $1.5-2.0 \mathrm{~cm}$ polyp along the greater curvature of the distal body of the stomach (fig. 1). Biopsy demonstrated intestinal metaplasia with patchy epithelial atypia, indefinite for dysplasia and mild chronic gastritis, however without evidence of adenocarcinoma. It was suggested that the gastritis be treated medically and a repeat biopsy be performed if clinical suspicion of neoplasm remained. Unfortunately the patient decided to delay her follow-up evaluation for one year.

One year after initial endoscopic biopsy the patient returned, experiencing intermittent episodes of flushing and abdominal bloating. Laboratory examination identified resolution of the anemia with hemoglobin of $13.4 \mathrm{~g} / \mathrm{dl}$, hematocrit of $39.1 \%$, and mean corpuscular volume of $94 \mathrm{fl}$. Endoscopic ultrasound was performed which demonstrated a gastric polypoid lesion emanating from the second mucosal layer with a superficial cystic structure noted within the submucosa (fig. 2). The muscularis propria was intact and no adenopathy was observed followed by endoscopic mucosal resection (fig. 3 ). The polyp from the body of the stomach measured $1.3 \times 1.2 \times 1.1 \mathrm{~cm}$ and displayed mixed adenocarcinoma and carcinoid with extension to the muscularis mucosae and submucosa. The specimen demonstrated antral type mucosa and intestinal metaplasia on a background of chronic gastritis. The specimen, indentified as an infiltrating epithelial neoplasm, demonstrated two morphologically separate components. One portion contained mucinous glands with clusters of cells displaying moderate to marked nuclear atypia most suggestive of intestinal adenocarcinoma. Intertwined with these mucinous glands were small clusters of cells with far less atypical nuclei with marked neuroendocrine appearance representing a focus of carcinoid tumor (fig. 4). The carcinoid portion of the tumor was relatively small and extended into the submucosa. Staining was positive for chromogranin A and synaptophysin and keratins: 40, 48, 50, 52, 54, 56.5, 58, 59, 64, 67 (fig. 5). The thiazine differential and immunoperoxidase stains were negative for Helicobacter pylori. MRI of the abdomen done with and without contrast showed no evidence of metastatic disease. Definitive treatment was accomplished by partial gastrectomy as the patient is doing well and approaching her two-year follow-up without evidence of disease or return of symptoms.

\section{Discussion}

Studies have found an association between gastric carcinoid tumors and autoimmune atrophic gastritis with hypergastrinemia [8]. It has been suggested that $H$. pylori, on a foundation of atrophic gastritis, may also be a contributing factor in the development of gastric carcinoid tumors [12]. Carcinoids have been identified in gastric tissue in association with hypergastrinemia-related syndromes, namely multiple endocrine neoplasia type 1 (Wermer syndrome) and Zollinger-Ellison syndrome as well as in patients without hypergastinemia $[6,8,12,13]$. It is suggested that effects of prolonged hypergastremic states predispose the mucosal cells to hyperplasia, especially in the setting of atrophic gastritis [13]. While carcinoids are generally more indolent in nature, they may demonstrate a greater degree of malignant potential if in occurrence with concomitant gastric adenocarcinoma [14]. According to Rindi et al., gastric carcinoid tumors can be classified into three distinct subtypes. Type I includes carcinoids that develop on a foundation of atrophic corporal gastritis, type II includes carcinoids associated with MEN type I syndrome or Zollinger-Ellison syndrome, and type III includes carcinoids with malignant features in the absence of hypergastrinemia [15], with type III being the mostly likely to undergo regional lymph node metastasis and therefore accompanied by the worst prognosis [8]. In our case the patient falls into type I, as evidenced by the background of atrophic gastritis seen on endoscopic and pathologic examination with subsequent MRI demonstrating no evidence for regional lymph node metastasis. Rindi's classification correlated with different survival rates in the three 
subgroups, with group I having the best overall prognosis in that series. Based on this, the treatment of isolated carcinoid tumors should proceed based on the subtype identified.

Many gastric adenocarcinomas have neuroendocrine components. Likewise, neuroendocrine tumors have demonstrated areas composed of adenocarcinomatous cells. These combinations have been described as 'composite' or mixed glandular-endocrine tumors [16]. At present, it is unclear whether the two cell types are due to proliferation of two distinct cell lines or rather a proliferation of a single line of pluripotent cellular precursor cells that divide into the two cells types [16].

Lewin [6] classifies the group of tumors demonstrating mixed epithelial and endocrine components into three subgroups. Group I consists of composite glandular-endocrine cell tumors, group II of amphicrine tumors and group III of collision tumors. Lewin classifies collision tumors as a subtype in which a carcinoid abuts an adjacent adenocarcinoma or adenoma with the two distinct cell types in juxtaposition to one another without evidence of intermixing. The group I classification allows for mixed cellular components without a fixed neuroendocrine to endocrine ratio [6]. In our case, the patients' pathology is most consistent with Lewin's group I classification. The reason for such differentiation accounts for the tumor's distinctive clinical behavior at particular anatomic locations and may serve as a guide to directing treatment. In our case, management was directed towards the larger and generally more aggressive adenocarcinoma component.

\section{Conclusion}

Atrophic gastritis as well as pernicious anemia have been found to be risk factors for the development of both gastric adenocarcinoma and carcinoid tumors [13]. In such patients, surveillance by endoscopic means can be employed to offset the increased risk of neoplastic development. Recently there is a trend to follow such patients with type I gastric carcinoids with surveillance endoscopy and to avoid surgical management. The study by Gladdy et al. [8] demonstrated no appreciable difference in mortality between patients followed by endoscopy when compared with surgical intervention. However, in patients with concurrent gastric adenocarcinoma, surgical resection is generally preferred. In this particular patient, management proceeded with surgical resection, however in cases where the tumor does not display submucosal invasion, endoscopic mucosal resection may serve as a definitive treatment. The decision to proceed with surgical resection was justified as the patient has no evidence of disease after one year of follow-up. As in our case, management should generally be directed towards the more aggressive and threatening component of the neoplasm to assure the best outcome.

\section{Disclosure Statement}

The authors have no disclosures or conflicts of interests. No funding was received for this paper.

\section{Consent}

Written informed consent was obtained from the patient and is available for review upon request. 


\begin{tabular}{|c|c|c|c|}
\hline Case & \begin{tabular}{|l} 
Case Rep Gastroenterol 2011;5:232-238 \\
DOl: 10.1159/000327986
\end{tabular} & $\begin{array}{l}\text { Published online: } \\
\text { April 15, } 2011\end{array}$ & \begin{tabular}{|l} 
O 2011 S. Karger AG, Basel \\
ISSN $1662-0631$ A, Bar \\
www.karger.com/crg
\end{tabular} \\
\hline
\end{tabular}

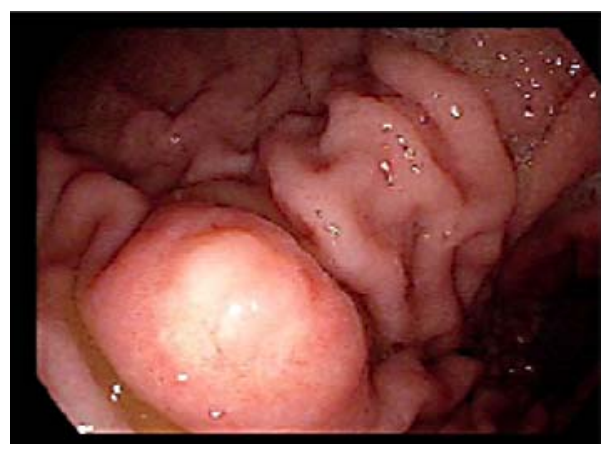

Fig. 1. Upper endoscopy showing a polypoid mass in the body of the stomach.

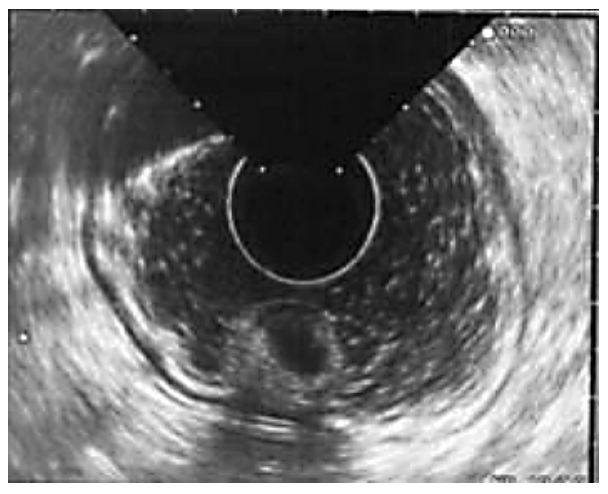

Fig. 2. Endoscopic ultrasound demonstrating the polypoid lesion with extension through the submucosal layer.

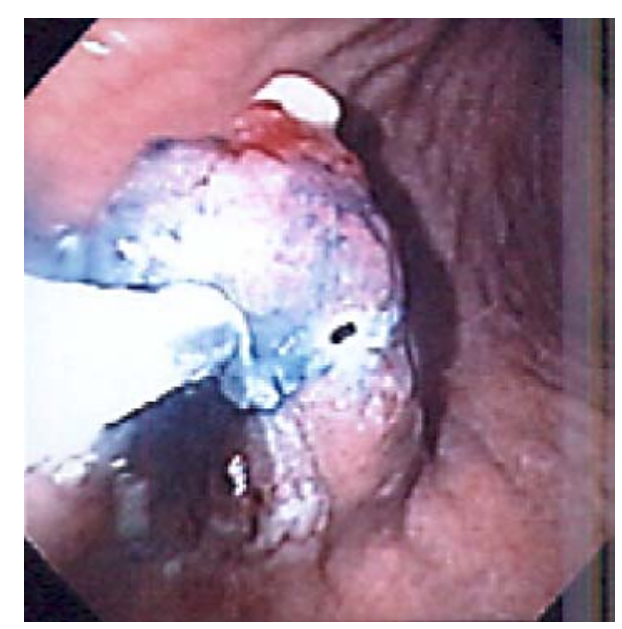

Fig. 3. Repeat endoscopy with resection of the polyp in the body of the stomach. 


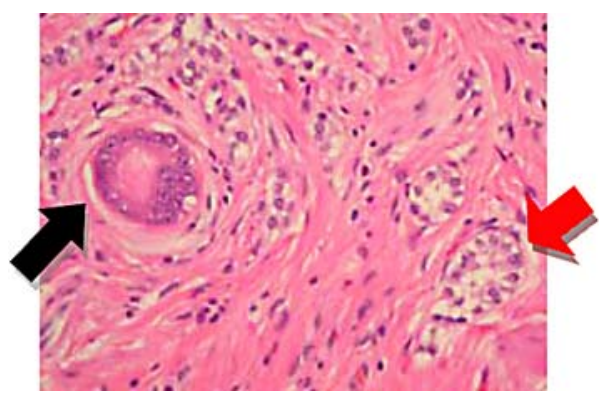

Fig. 4. Hematoxylin and eosin staining of the polyp demonstrating glandular tissue (adenocarcinoma) (black arrow) and foci of endocrine cells (carcinoid) (red arrow).

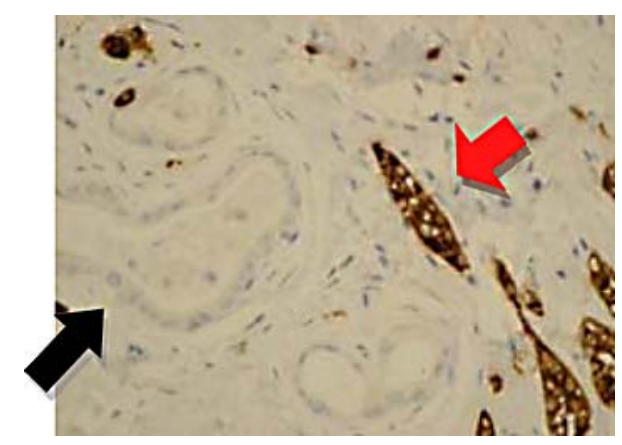

Fig. 5. Immunoperoxidase stain with positivity for synaptophysin (red arrow) in carcinoid and negativity in adenocarcinoma (black arrow).

\section{References}

1 Dicken BJ, Bigam DL, Cass C, Mackey JR, Joy AA, Hamilton SM: Gastric adenocarcinoma: review and considerations for future directions. Ann Surg 2005;241:27-39.

-2 Pidoto RR, Piquard A, Fama F, Marc OS: Lymph node metastasis from gastric carcinoid tumors occurring concomitantly with gastric adenocarcinomas and atrophic gastritis. J Gastrointest Surg 2006;10:402-406.

-3 Soga J: Gastric carcinoids: a statistical evaluation of 1,094 cases collected from the literature. Surg Today 1997;27:892-901.

-4 Shinohara T, Ohyama S, Nagano H, Amaoka N, Ohta K, Matsubara T, Yamaguchi T, Yanagisawa A, Kato Y, Muto T: Minute gastric carcinoid tumor with regional lymph node metastasis. Gastric Cancer 2003;6:262-266.

5 Borch K: Atrophic gastritis and gastric carcinoid tumours. Ann Med 1989;21:291-297.

6 Lewin K: Carcinoid tumors and mixed (composite) glandular-endocrine cell carcinomas. Am J Surg Pathol 1987;11(suppl 1):71-86.

7 Bi R, Sheng W, Wang J: Collision tumor of the stomach: gastric adenocarcinoma intermixed with gastrointestinal stromal tumor. Pathol Int 2009;59:880-883.

-8 Gladdy RA, Strong VE, Coit D, Allen PJ, Gerdes H, Shia J, Klimstra DS, Brennan MF, Tang LH: Defining surgical indications for type I gastric carcinoid tumor. Ann Surg Oncol 2009;16:3154-3160.

9 Borch K: Epidemiologic, clinicopathologic, and economic aspects of gastroscopic screening of patients with pernicious anemia. Scand J Gastroenterol 1986;21:21-30.

10 Adhikari D, Conte C, Eskreis D, Urmacher C, Kahn E: Combined adenocarcinoma and carcinoid tumor in atrophic gastritis. Ann Clin Lab Sci 2002;32:422-427. 
-11 Caruso ML, Pilato FP, D’Adda T, Baggi MT, Fucci L, Valentini AM, Lacatena M, Bordi C: Composite carcinoid-adenocarcinoma of the stomach associated with multiple gastric carcinoids and nonantral gastric atrophy. Cancer 1989;64:1534-1539.

12 Solcia E, Rindi G, Fiocca R, Villani L, Buffa R, Ambrosiani L, Capella C: Distinct patterns of chronic gastritis associated with carcinoid and cancer and their role in tumorigenesis. Yale J Biol Med 1992;65:793-804.

13 Borch K, Renvall H, Lieberg G, Andersen BN: Relations between circulating gastrin and endocrine cell proliferation in the atrophic gastric fundic mucosa. Scand J Gastroenterol 1986;21:357-363.

-14 Nagaoka S, Toyoshima H, Bandoh T, Isoyama T, Kasahara D, Kusafuka K, Takemura T, Tanaka N: Composite carcinoid-adenocarcinoma tumor of the stomach: report of a case. Surg Today 1996;26:184-188.

15 Rindi G, Bordi C, Rappel S, La Rosa S, Stolte M, Solcia E: Gastric carcinoids and neuroendocrine carcinomas: pathogenesis, pathology and behavior. World J Surg 1996;20:168-172.

16 Lee EJ, Park SM, Maeng L, Lee A, Kim KM: Composite glandular-endocrine cell carcinomas of the stomach: clinicopathologic and methylation study. APMIS 2005;113:569-576. 